\title{
Magnetic/gold nanoparticle functionalized biocompatible microcapsules with sensitivity to laser irradiation
}

\author{
Dmitry A. Gorin, ${ }^{a}$ Sergey A. Portnov, ${ }^{a}$ Olga A. Inozemtseva, ${ }^{a}$ Zofia Luklinska, ${ }^{c}$ \\ Alexey M. Yashchenok, ${ }^{a}$ Anton M. Pavlov, ${ }^{a}$ Andre G. Skirtach, ${ }^{b}$ \\ Helmuth Möhwald $^{b}$ and Gleb B. Sukhorukov ${ }^{c}$
}

Received 9th June 2008, Accepted 26th August 2008

First published as an Advance Article on the web 14th October 2008

DOI: $10.1039 /$ b809696a

\begin{abstract}
Nanocomposite microcapsules with both gold and magnetite nanoparticles in the shell were prepared in a layer-by-layer procedure using biocompatible polyelectrolytes and nanoparticles. The process of a nanocomposite multilayer formation was investigated using a quartz crystal microbalance (QCM). In addition, nanocomposite microcapsules were characterized by atomic force microscopy (AFM), transmission electron microscopy (TEM) and energy-dispersive X-ray spectroscopy (EDX). It is found that the amount of adsorbed nanoparticles is similar for nanoparticles of various sizes, while the concentration of gold nanoparticles in the shell is higher for smaller nanoparticles. Adsorption of gold nanoparticles is found to be more effective than adsorption of magnetic nanoparticles. Multifunctionality of microcapsules is manifested by dual: magnetic and optical responses. Iron oxide nanoparticles embedded in the microcapsule shell allowed for control over capsules positioning by external magnetic fields. Furthermore, the nanocomposite microcapsules could be opened by laser irradiation; these results are of interest for medical and biological applications.
\end{abstract}

\section{Introduction}

A novel approach to treatment of certain medical conditions involves the development of special drug delivery systems with dose controlled abilities. However, new effective low toxicity medicines are expensive. Another method known as encapsulation can use established drugs, decreasing their toxicity, lowering the overall production cost and retaining the drug effectiveness in treatment of specific diseases. One prominent encapsulation method is through the use of layer-by-layer (LbL) adsorption. ${ }^{1-3}$ It can be equally used for the encapsulation of solid as well as liquid substances. ${ }^{4-8}$ The LbL assembly method is based on the bottom-up principle used in the fabrication of composite materials, ${ }^{9}$ which makes it possible to achieve a significant control over the physical properties of nanodimensional shells ${ }^{10,11}$ or planar layers. ${ }^{12-16}$

A study of the control of microcapsule properties by varying shell composition and physicochemical parameters of the surrounding medium for encapsulation of substances and their controlled release was already published. ${ }^{17}$ However, many applications also require remote control over the permeability of the microcapsule shell. This can be done by light or laser irradiation, ${ }^{18-23}$ alternating magnetic field, ${ }^{24}$ microwave radiation $^{25}$ or ultrasound. ${ }^{26-28}$ Considerable progress was achieved in the use of laser irradiation for microcapsule opening. ${ }^{22,23}$

${ }^{a}$ Faculty of Nano- and Biomedical Technologies, Saratov State University, Saratov, 410012, Russia.E-mail: gorinda@info.sgu.ru, gorinda@mail.ru; Tel: +78452511181

${ }^{b}$ Max-Planck Institute of Colloids and Interfaces, D-14476 Golm/ Potsdam, Germany

${ }^{c}$ Department of Materials, Queen Mary University of London, London, UK E1 4NS
Usually, metal nanoparticles ${ }^{19-23}$ or dyes ${ }^{19}$ are used to provide microcapsule shell sensitivity to laser radiation. Our research has recently demonstrated that it is possible to release encapsulated materials inside living cells from laser-sensitive capsules. $^{22}$ It was also established, that the use of aggregates composed of nanoparticles decreases the laser intensity, necessary for microcapsule opening and release of encapsulated substances. $^{23}$

Some hurdles in use of lasers for microcapsules opening are connected with a local effect of laser irradiation. Iron oxide nanoparticles present in the capsule shell can be used to concentrate and transfer the microcapsules by magnetic field ${ }^{10}$ and to influence the distribution of gold nanoparticles in the nanocomposite shell during their preparation. Thus, the fabrication of biocompatible microcapsules containing both iron oxide and gold nanoparticles is a very important task from a biomedical application point of view. Also, for application in medicine one needs to use longer wavelengths that penetrate tissue deeper. $^{22}$

In this work, adsorption of polyelectrolyte molecules and nanoparticles was studied on the planar substrate by the QCM method. Nanocomposite microcapsule shells composed of iron oxide together with gold nanoparticles in the shell were therefore prepared. The overall concentration of the nanoparticles was adjusted by varying the number of adsorbed nanoparticle layers in the composite shell containing gold nanoparticles. The microcapsules were characterized by AFM and TEM methods. The effect of a magnetic field on the microcapsule concentration in a suspension was also established, together with the sensitivity of the microcapsules to laser irradiation (Fig. 1). 


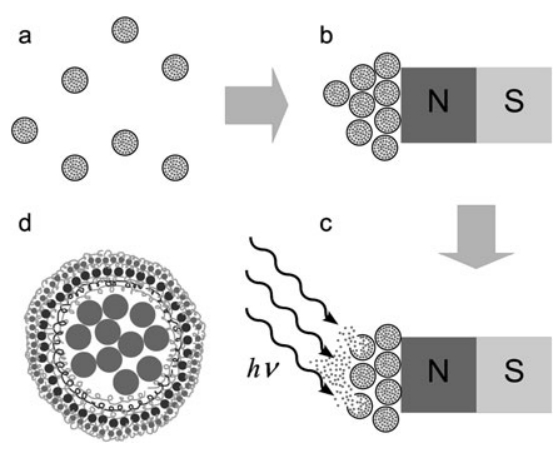

Fig. 1 Illustration of application of photosensitive magnetic microcapsules for drug delivery applications: a - capsules before any excitation; b-concentration of capsules caused by magnetic field; c - destruction of concentrated capsules followed with capsulated substance release; $d$-illustration of shell structure needed to achieve sensitivity to laser radiation and magnetic field. Large grey circles inside the capsule shell in Fig. 1d illustrates capsulated substance, medium-sized dark-grey circles are magnetic iron oxide nanoparticles and small light-grey circles represents gold plasmon-resonance particles.

\section{Experimental}

\section{Materials}

The poly(L-argininine) (PArg, MW 15-70 kDa), dextrane sulfate sodium salt (DS, MW $100 \mathrm{kDa}$, Fluka), polyethylenimine (PEI, MW 600-1000 kDa), disodium ethylenediaminetetraacetate (EDTA), hydrochloric acid, calcium chloride dehydrate $\left(\mathrm{CaCl}_{2} \cdot 2 \mathrm{H}_{2} \mathrm{O}\right)$, sodium carbonate $\left(\mathrm{Na}_{2} \mathrm{CO}_{3}\right)$, water suspension of iron oxide nanoparticles stabilized by citric acid $\left(\mathrm{Fe}_{3} \mathrm{O}_{4}\right)$ less than $50 \mathrm{~nm}$ in size, gold colloids (Au5, Au10, Au20) with particle diameters of 5, 10 and $20 \mathrm{~nm}$ were purchased from Sigma-Aldrich. The water used in all experiments was prepared by a Purelab UHQ purification system (ELGA, LabWater Global Operation, UK) and had a resistivity higher than $18 \mathrm{M} \Omega \mathrm{cm}$.

\section{Preparation of $\mathrm{CaCO}_{3}$ microparticles}

Calcium carbonate templates were fabricated as described previously. $^{29}$ The process was initiated by rapid mixing of equal volumes of $0.33 \mathrm{M}$ water solutions of $\mathrm{CaCl}_{2}$ and $\mathrm{Na}_{2} \mathrm{CO}_{3}$ at room temperature. After intense agitation with a magnetic stirrer for 30 seconds, the precipitate was obtained by sedimentation and rinsed with water three times. The sedimentation of $\mathrm{CaCO}_{3}$ microparticles was performed by centrifugation at $1000 \mathrm{rpm}$ for $1 \mathrm{~min}$. This procedure formed a water suspension containing spherical $\mathrm{CaCO}_{3}$ microparticles with an average diameter ranging from 3 to $5 \mu \mathrm{m}$.

\section{Fabrication of planar LbL assemblies and quartz crystal microbalance (QCM) measurements}

The automated setup POLYION-1M was used in the preparation of polyelectrolyte films on the surface of the quartz crystal resonator. Formation of the layer by layer assembly using this setup has already been reported. ${ }^{30}$ The following ingredients were used: $1 \mathrm{mg} \mathrm{ml}^{-1}$ PArg and DS solutions in $0.15 \mathrm{M} \mathrm{NaCl}$ and also water suspensions of iron oxide and gold nanoparticles. The iron oxide nanoparticle suspension was diluted 50 times from its initial concentration. Gold colloids (average size $5 \mathrm{~nm}$ (Au5), $10 \mathrm{~nm}$ (Au10) and $20 \mathrm{~nm}$ (Au20)) were used without dilution. A PEI layer ( $2 \mathrm{mg} \mathrm{ml}^{-1}$ water solution) acted as a precursor. The process of adsorption from solution lasted 15 minutes. For QCM measurements an AT-cut $8.86 \mathrm{MHz}$ quartz crystal electrode was employed as a substrate for the LbL deposition. Surfaces of the resonators were rinsed with deionized water before use. Quartz crystal resonators were washed three times with deionised water after each adsorption step. Planar films of the following structures: $\mathrm{PEI} /(\mathrm{DS} / \mathrm{PArg})_{5} / \mathrm{DS}$, $\mathrm{PEI} /\left(\mathrm{Fe}_{3} \mathrm{O}_{4} / \mathrm{PArg}\right)_{5} / \mathrm{Fe}_{3} \mathrm{O}_{4}, \quad \mathrm{PEI} /(\mathrm{Au} 5 / \mathrm{PArg})_{5} / \mathrm{Au} 5, \quad \mathrm{PEI} /$ (Au10/PArg) $5 / \mathrm{Au} 10, \mathrm{PEI} /(\mathrm{Au} 20 / \mathrm{PArg})_{5} / \mathrm{Au} 20$ were prepared on the surface of the quartz crystal resonators. Polyelectrolyte and nanoparticle layers were dried for 10 minutes at room temperature in air between deposition and the frequency measurement, in order to provide reproducible data for the QCM technique.

\section{Capsule preparation}

Calcium carbonate microparticles with average core diameter of $4 \pm 1 \mu \mathrm{m}$ were used as a template for fabrication of the nanocomposite shells. The first polyelectrolyte layer was made by adsorption of the positively charged PArg from $1 \mathrm{mg} \mathrm{mL}^{-1}$ solution in $0.15 \mathrm{M} \mathrm{NaCl}$ (15 min of incubation and shaking) on $\mathrm{CaCO}_{3}$ microparticles dispersed in this solution. The second layer was prepared by absorption of the negatively charged DS from $1 \mathrm{mg} \mathrm{ml}^{-1}$ solution in $0.15 \mathrm{M} \mathrm{NaCl}(15 \mathrm{~min}$ of incubation and shaking). The core/polyelectrolyte particles were washed three times with deionised water after each adsorption step. Adsorption of the iron oxide nanoparticles occurred by immersion in diluted water suspension in a ratio 1:50. Gold colloids (average size $5 \mathrm{~nm}$ (Au5), $10 \mathrm{~nm}$ (Au10) and $20 \mathrm{~nm}$ (Au20)) were used without dilution. The calcium carbonate cores were dissolved in ethylenediamine tetraacetic acid (EDTA) for 30 minutes, then centrifuged and rinsed 3 times with EDTA and then 3 times using water. Three types of the microcapsule shells were manufactured. The first type was composed of initial microcapsules without any nanoparticles in the shell, (PArg/DS) 6 named as "Sample 0". The second type consisted of one layer of iron oxide nanoparticles and two layers of gold nanoparticles with mean diameters of 5, 10 and $20 \mathrm{~nm}$ (samples 1-5, 1-10, 1-20, respectively): (PArg/DS)(PArg/ $\left./ \mathrm{Fe}_{3} \mathrm{O}_{4}\right)(\mathrm{PArg} / \mathrm{Au} 5)_{2}(\mathrm{PArg} / \mathrm{DS})$; (PArg/DS)(PArg/Fe $\left.\mathrm{O}_{4}\right)$ (PArg/Au 10) $)_{2}$ (PArg/DS); (PArg/DS)$\left(\mathrm{PArg} / \mathrm{Fe}_{3} \mathrm{O}_{4}\right) \quad(\mathrm{PArg} / \mathrm{Au} 20)_{2}(\mathrm{PArg} / \mathrm{DS})$. The third type contained two layers of iron oxide nanoparticles and one layer of gold nanoparticles with 5, 10 and $20 \mathrm{~nm}$ mean diameters (samples 2-5, 2-10, 2-20, respectively): (PArg/DS)(PArg/ $\left.\mathrm{Fe}_{3} \mathrm{O}_{4}\right)_{2-}$ (PArg/Au5)(PArg/DS); (PArg/DS)(PArg/Fe $\left./ \mathrm{O}_{4}\right)_{2}(\mathrm{PArg} / \mathrm{Au} 10)-$ (PArg/DS); (PArg/DS)(PArg/Fe $\left.\mathrm{O}_{4}\right)_{2}(\mathrm{PArg} / \mathrm{Au} 20)(\mathrm{PArg} / \mathrm{DS})$.

\section{$\zeta$-Potential measurements}

The $\zeta$-potential of nanoparticle suspensions was measured by a Zetasizer Nano-Z (Malvern Instruments Ltd, UK). Overall, 10 measurements were performed. Each result represents an average of three subsequent runs. 


\section{Optical microscopy}

Transmission optical and scanning confocal images of the capsules were obtained using INTEGRA Spectra scanning probe nanolaboratory (NT-MDT, Russia). Measurements were performed using $100 \times$ objective lens installed in the INTEGRA base. Optical photos were taken from video signal produced by the camera mounted in the transmission part of the optical system. Confocal images were taken under irradiation by the $473 \mathrm{~nm}$ semiconductor laser.

\section{Transmission electron microscopy (TEM)}

Samples for TEM examination were prepared by placing a drop of water suspension containing microcapsules directly onto the carbon coated TEM copper grids (Agar Scientific) and left to dry. Bright field TEM images were obtained in a high resolution electron microscope Jeol JEM-2010 (Jeol, Japan) operated at an acceleration voltage of $200 \mathrm{kV}$.

\section{Energy-dispersive X-ray spectroscopy (EDS)}

The TEM specimens were also analyzed by energy-dispersive $\mathrm{X}$-ray spectroscopy, in order to determine the chemical composition of the nanocomposite microcapsules. In this method, the X-ray intensity of the elements is plotted against their characteristic energy. Quantitative analysis was performed using INCA, Energy 300 system (Oxford Instruments, UK).

\section{Atomic force microscopy}

The AFM images of dried microcapsules were recorded by a Solver Bio AFM (NT-MDT, Russia) operating in tapping mode. The AFM tips having a resonance frequency of about $302-354 \mathrm{kHz}$ and stiffness of about $25-42 \mathrm{~N} \mathrm{~m}^{-1}$ were purchased from NT-MDT. The samples were prepared by drying a drop of the microcapsule water suspension on a mica substrate at room temperature.

\section{Remote activation of microcapsules}

Remote activation of microcapsules was conducted by the method described previously. ${ }^{19,31}$

In this particular case, a pulsed laser operating at wavelength $840 \mathrm{~nm}$, pulse duration $700 \mathrm{ps}$, was coupled into the optical system, and imaging was taken in the transmission mode.

\section{Results and discussion}

\section{$\zeta$-Potential of gold and iron oxide nanoparticles}

The $\zeta$-potential of the gold nanoparticles did not depend on their size and was found as $-49.4 \pm 9.8 \mathrm{mV},-42.5 \pm 5.8 \mathrm{mV}$ and $-48.8 \pm 8.7 \mathrm{mV}$ for 5,10 and $20 \mathrm{~nm}$ particles, respectively. The $\zeta$-potential for iron oxide nanoparticles measured $-12.5 \pm 4.0 \mathrm{mV}$.

\section{QCM study of the planar nanocomposite films by LbL adsorption}

Investigations of the LbL assembly on the planar substrate ${ }^{13,32}$ using the quartz crystal resonators as substrates were performed by the QCM method. ${ }^{33}$ The frequency change of the resonator with the number of polyelectrolyte and nanoparticle

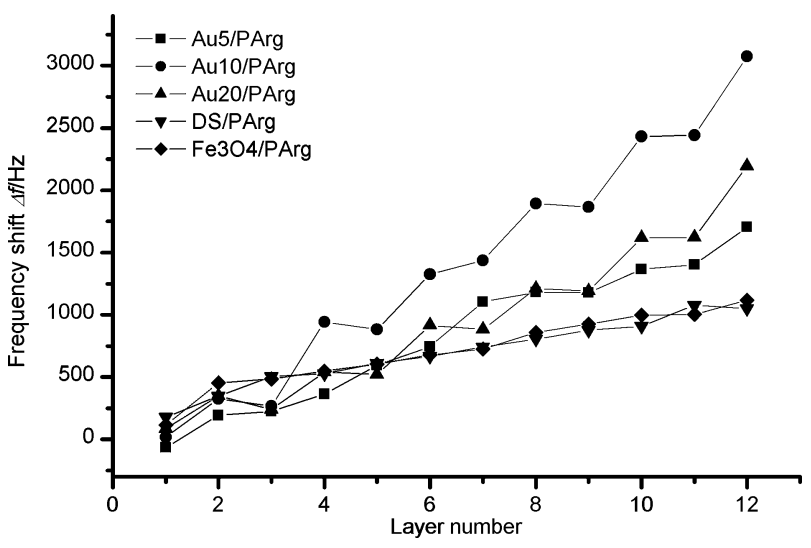

Fig. 2 QCM frequency changes with the formation of the multilayer films; first layer was PEI. The odd layer numbers show the deposition of polyarginine, and the even numbers correspond to the deposition of the nanoparticles.

layers is presented in Fig. 2. One layer of PEI (600000 $1000000 \mathrm{MW}$ ) was used as a precursor ${ }^{13,34,35}$ for all samples. It was found that a high molecular weight of PEI improved the adsorption capabilities of the substrate for the LbL assembly deposition. ${ }^{34,35}$ The data in Fig. 2 represent a typical frequency relationship for the layer by layer assembly. It can be concluded from these data that adsorption of the gold nanoparticles was more effective, in comparison to the iron oxide nanoparticles or anionic polyelectrolytes. The adsorption density was found to depend on the nanoparticle size. Maximum frequency change was achieved for gold nanoparticles of $10 \mathrm{~nm}$ size. To explain this fact one needs to mention that frequency change depends on the mass of the adsorbed nanoparticles. The latter, in turn, is the product of the nanoparticle concentration and the mass of a single nanoparticle. Assuming that the density of the nanoparticle material does not depend on nanoparticle size, the concentration of the $10 \mathrm{~nm}$ nanoparticles could be eight times lower than for the $5 \mathrm{~nm}$ size particles having the same total mass. However, in our study, the average frequency change due to adsorption of the $10 \mathrm{~nm}$ nanoparticles was only two times higher than for the $5 \mathrm{~nm}$ nanoparticles, meaning that the concentration of the $5 \mathrm{~nm}$ nanoparticles was higher than the concentration of the $10 \mathrm{~nm}$ particles. Overall, a decrease in the nanoparticle size led to an increase in an adsorption density. The mass transfer of the magnetite particle layer was comparable with the mass transfer of the polyelectrolyte layer. As far as iron oxide density is higher than polyelectrolyte one and nanoparticle average diameter is higher than known polyelectrolyte layer thickness, a dense nanoparticle layer doesn't get formed. Therefore, our results suggest that the iron oxide nanoparticles in the microcapsule shell rather form the sparse layer or can have heterogeneous distribution, hence some agglomerates of the particles could form in the shell.

\section{Optical microscopy study}

Optical photos (Fig. 3a) clearly show the existence of the capsules and the shape of their shells. Capsules in all samples had quite a similar look under the optical microscope. The low contrast of the optical images is connected with capsule shells 


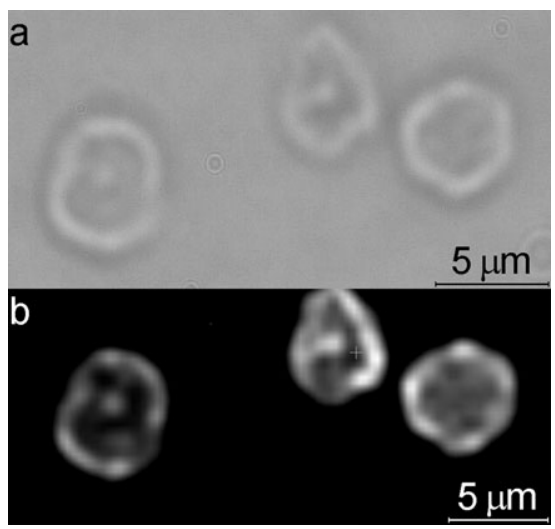

Fig. 3 Optical (a) and confocal (b) images of sample 1-10 capsules. A $473 \mathrm{~nm}$ laser was used to obtain image (b).

thickness which is lower than resolution of the optical system. However, confocal laser scanning images demonstrate better contrast. To obtain the contrast images two spectra obtained inside and outside the capsule were compared. A spectral band was found where scattered signal intensity taken inside the capsules was stronger than one outside the capsule. Confocal image (Fig. 3) represents the spatial distribution of the average signal value inside this band. One can clearly see the grid on this image which is in good correlation with scan step size.

\section{AFM study of microcapsule shells}

To obtain images of higher resolution AFM method was used. It provided all necessary information about capsules shape, size and shell thickness. Fig. 4 shows the example AFM image of sample 2-5 obtained in tapping mode. Granular structure is clearly seen. The capsule shell thickness was calculated from the height profiles of 15 microcapsules. Fig. 3 shows the relationship between the shell structure and the microcapsule shell thickness. It was observed that the thickness of the nanocomposite shell does not depend on the size of the gold nanoparticles. This fact takes place due to the homogeneous distribution of the gold nanoparticles in a microcapsule shell because of the penetration of the gold nanoparticles into the polyelectrolyte layers. Increasing the number of the iron oxide nanoparticle layers led to an increase in the nanocomposite shell thickness (Fig. 5). These experimental data correlate well with the previously published results. ${ }^{10}$ A significant increase in the shell thickness was likely caused by the agglomeration of

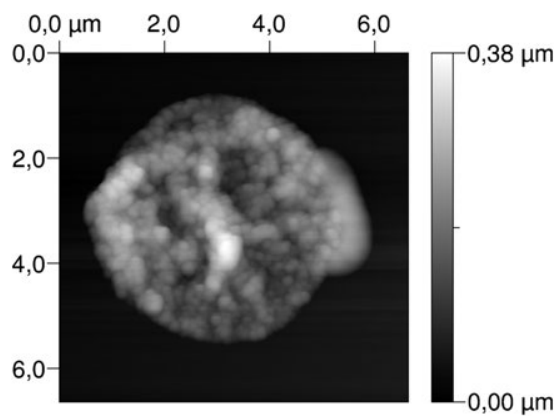

Fig. 4 AFM image of sample 2-5 capsule obtained in tapping mode. Granular structure is clearly seen.

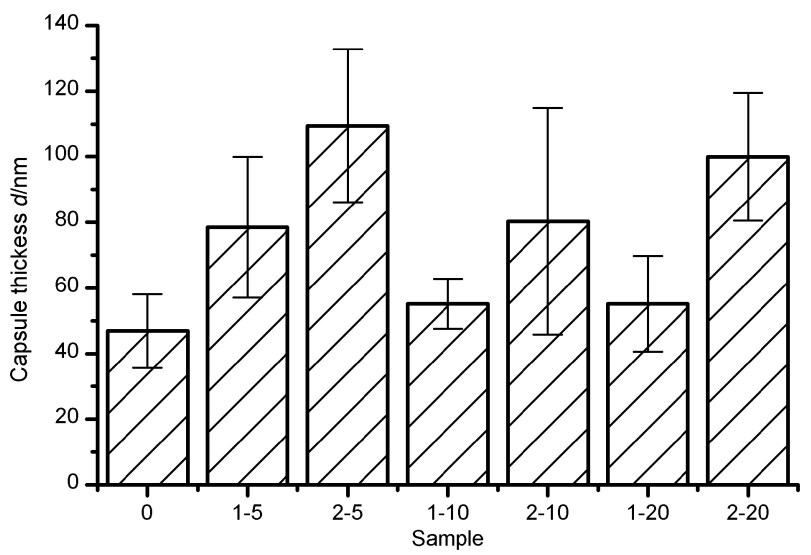

Fig. 5 AFM-measured thickness of the dried and collapsed microcapsules shells. The values are doubled shell thicknesses.

the iron oxide nanoparticles, leading to an increase in the nanoparticle volume fraction present in the microcapsule shell. The other study also established that the agglomerates formation is typical for the magnetic nanoparticles. ${ }^{13,24}$

\section{TEM and EDS examination of the nanocomposite shell}

TEM examination of the samples revealed a homogeneous distribution of the gold nanoparticles in the nanocomposite shell (Fig. 6). The TEM images were used to evaluate the concentration of the gold nanoparticles $\left(C_{\mathrm{Au}}\right)$ and the filling factor $\left(F F_{\mathrm{Au}}\right)$ (Table 1). These parameters were found to increase as the nanoparticle size decreased. This finding is in agreement with the data obtained by the QCM and AFM methods, and can be explained as follows. First, the Coulomb interaction force between the charges of polyelectrolyte layers and the nanoparticles is proportional to the contact area between them. The contact areas can be calculated as a surface area of the spherical cap $S=2 \pi r d$, where $r$-nanoparticle radius and $d-$ the layer thickness (Fig. 7). Since the adhesion energy is expected to scale with the contact area $2 \pi r d$ we have to postulate a force for removal which scales with $r$ with a higher power that one. This force may result from convection since the shear force is expected to scale with $r$ and also the Stokes friction scales with $r$. The probability of the nanoparticle desorption depends on the ratio $K$ between the contact area and the total surface area of the nanoparticle. This ratio can be estimated as $K=(2 \pi r d) /\left(4 \pi r^{2}\right)=d /(2 r)$. The $K$ values were calculated as $0.2,0.1$ and 0.05 for 5,10 and $20 \mathrm{~nm}$ nanoparticles, respectively, for the $2 \mathrm{~nm}$ polyelectrolyte layer thickness. Hence the larger nanoparticles would be removed easier than the smaller ones.

An increase in nanoparticle concentration leads to increase of the repulsive force acting between them which is known to be dependent on their charges and interparticle distance. The total charge of a nanoparticle is proportional to its surface area and the surface charge density. The latter one is connected to the $\zeta$-potential of the nanoparticles, which was found to be almost similar for all gold nanoparticle suspensions used in this study. Thus, the repulsive force is proportional to the square of the nanoparticle surface area or to the fourth power of their radius, assuming that the distance between the 


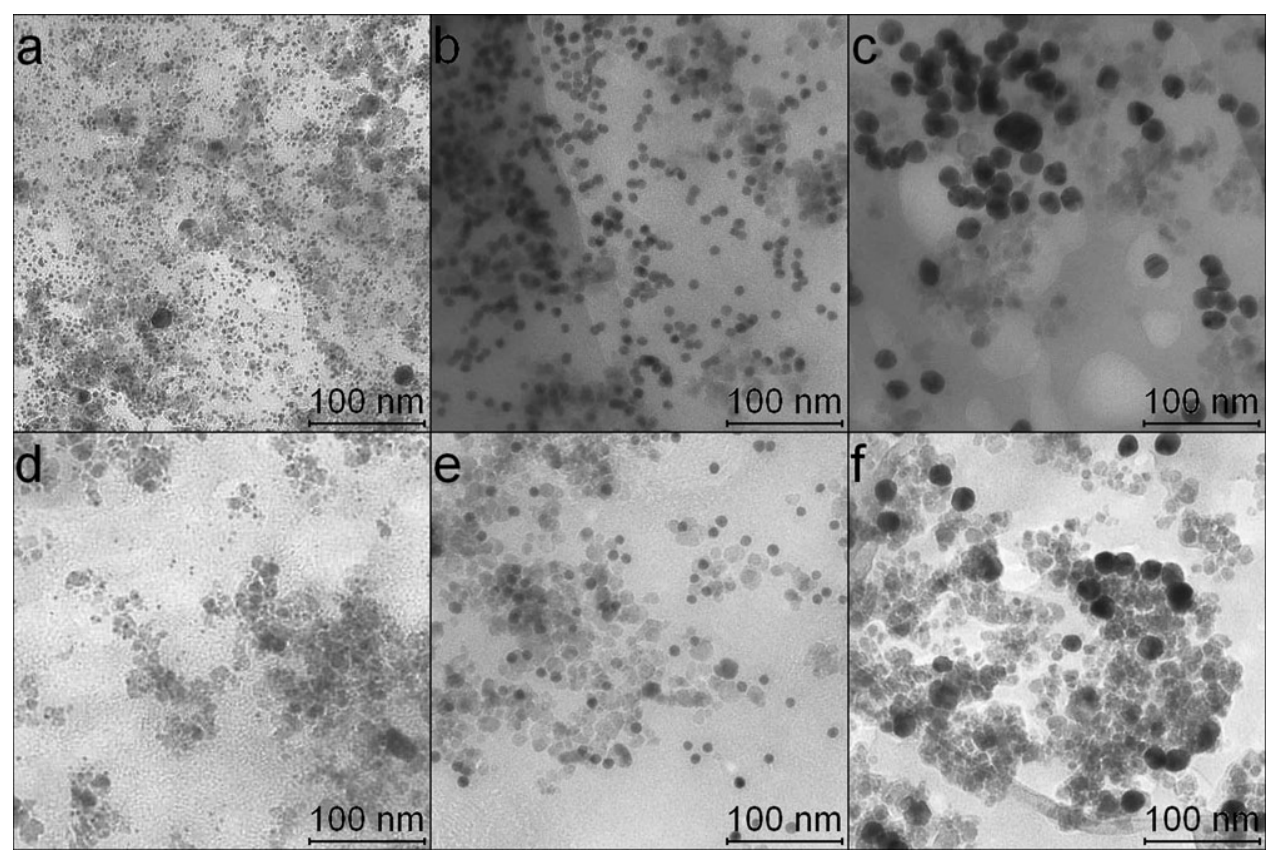

Fig. 6 TEM images of the microcapsules shells (fragments): a—sample 1-5; b-sample 1-10; c-sample 1-20; d-sample 2-5; e-sample 2-10; f-sample 2-20.

Table 1 Structures and chemical composition of nanocomposite microcapsule shells, number of the iron oxide nanoparticles layers $N_{\mathrm{Fe} 3 \mathrm{O} 4}$, number of gold nanoparticles layers $N_{\mathrm{Au}}$, concentration of the gold nanoparticles $C_{\mathrm{Au}}$, fill factor of the nanocomposite $F F_{\mathrm{Au}}$

\begin{tabular}{|c|c|c|c|c|}
\hline Number, and structures of the samples & $N_{\mathrm{Fe} 3 \mathrm{O} 4}$ & $N_{\mathrm{Au}}$ & $C_{\mathrm{Au}} / \mu \mathrm{m}^{-2}$ & $F F_{\text {Au }}(\%)$ \\
\hline $1-5,(\mathrm{PArg} / \mathrm{DS})\left(\mathrm{PArg} / \mathrm{Fe}_{3} \mathrm{O}_{4}\right)(\mathrm{Parg} / \mathrm{Au} 5)_{2}(\mathrm{PArg} / \mathrm{DS})$ & 1 & 2 & $33 \times 10^{2}$ & 26 \\
\hline $1-10,(\mathrm{PArg} / \mathrm{DS})\left(\mathrm{PArg} / \mathrm{Fe}_{3} \mathrm{O}_{4}\right)(\mathrm{Parg} / \mathrm{Au} 10)_{2}(\mathrm{PArg} / \mathrm{DS})$ & 1 & 2 & $4.3 \times 10^{2}$ & 14 \\
\hline $1-20,(\mathrm{PArg} / \mathrm{DS})\left(\mathrm{PArg} / \mathrm{Fe}_{3} \mathrm{O}_{4}\right)(\mathrm{Parg} / \mathrm{Au} 20)_{2}(\mathrm{PArg} / \mathrm{DS})$ & 1 & 2 & $2.6 \times 10^{2}$ & 3 \\
\hline $2-5,(\mathrm{PArg} / \mathrm{DS})\left(\mathrm{PArg} / \mathrm{Fe}_{3} \mathrm{O}_{4}\right)_{2}(\mathrm{Parg} / \mathrm{Au} 5)(\mathrm{PArg} / \mathrm{DS})$ & 2 & 1 & $13 \times 10^{2}$ & 10 \\
\hline $2-10,(\mathrm{PArg} / \mathrm{DS})\left(\mathrm{PArg} / \mathrm{Fe}_{3} \mathrm{O}_{4}\right)_{2}(\mathrm{Parg} / \mathrm{Au} 10)(\mathrm{PArg} / \mathrm{DS})$ & 2 & 1 & $2.3 \times 10^{2}$ & 7 \\
\hline $2-20,(\mathrm{PArg} / \mathrm{DS})\left(\mathrm{PArg} / \mathrm{Fe}_{3} \mathrm{O}_{4}\right)_{2}(\mathrm{Parg} / \mathrm{Au} 20)(\mathrm{PArg} / \mathrm{DS})$ & 2 & 1 & $1.2 \times 10^{2}$ & 1 \\
\hline
\end{tabular}

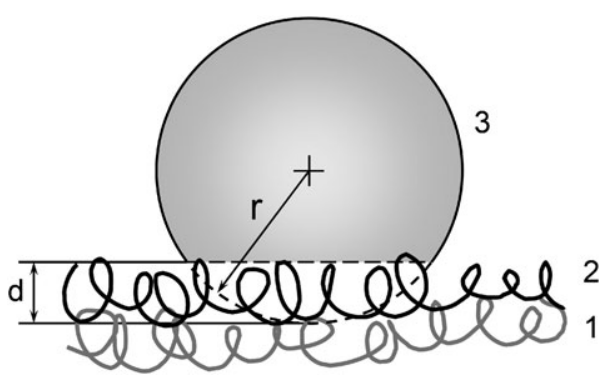

Fig. 7 Schematic illustration of the gold nanoparticle penetration into the polyelectrolyte layer of the microcapsule shell. The dependence of the gold nanoparticle concentration on its size is displayed: $d$-thickness of the polyelectrolyte layers, $r$-radius of the gold particles.

separate particles is constant. As a consequence, the probability of nanoparticle adsorption onto the surface of the polyelectrolyte layer will be lower for the nanoparticles of a larger diameter giving rise to a sparse distribution of nanoparticles.

Data presented in Table 1 show the dependence of the gold nanoparticle concentration $C_{\mathrm{Au}}$, and the fill factor of the nanocomposite surface $F F_{\mathrm{Au}}$ on the nanoparticle layer number
$N_{\mathrm{x}}$. The calculation shows that the fill factor $F F_{\mathrm{Au}}$ (evaluated in percentages) is proportional to the nanoparticle concentration $C_{\mathrm{Au}}$. Also, an increase in the number of the nanoparticle layers led to its higher concentration in the nanoparticle shell.

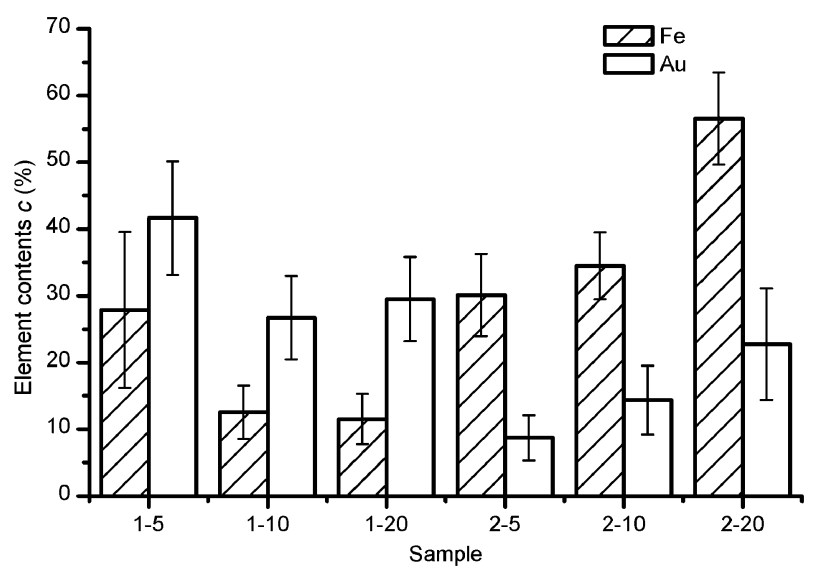

Fig. 8 EDS quantitative analysis of gold and iron in the capsules shells. Sample " 0 " is not included because of its insignificant concentrations of iron and gold. 
The EDS spectra (Fig. 8) of the nanocomposite shells agreed with the results obtained from the evaluation of the concentration of the nanoparticles using TEM images. Increasing the number of the nanoparticle layers led to an increase of the mass concentration. The maximum quantity of the gold nanoparticles in the microcapsule shell composed of two layers was observed for the $5 \mathrm{~nm}$ size. In the case of one gold nanoparticle layer, the data show similar trends to those described earlier in this paper for the QCM results.

\section{Effect of magnetic field on the nanocomposite microcapsule suspension}

A constant magnetic field interacts with nanocomposite shells through the magnetic nanoparticles embedded in them. ${ }^{36}$ This interaction causes microcapsule movement from places with low to those with high magnetic field strength. Thus, a local capsule concentration increase is achieved by means of a magnetic field. Thus, microcapsules can be concentrated and manipulated by the magnetic field, Fig. 9. Two transparent plastic cuvettes were filled with microcapsules suspensions and placed next to each other. At the beginning of the experiment microcapsules distribution was homogeneous in both cuvettes. Subsequently, all capsules were attracted to the side of the cuvette located next to the magnet. The residual liquid became transparent. Color of suspension in the right cuvette remained unchanged. Any distribution of color was not observed which means that capsules distribution change in the left cuvette was caused by magnetic field. Thus we have shown the possibility of control the local concentration of capsules containing gold and magnetite nanoparticles using magnetic field. It was established that inclusion of only one layer is enough to achieve capsule sensitivity to a magnetic field.

\section{Effect of laser irradiation on the nanocomposite shells}

The experiment in Fig. 10 clearly shows that laser irradiation can be used to open microcapsules. In these experiments, the capsules was first positioned in the field of view, Fig. 10a, at the location of the laser beam. Then was switched on, Fig. 10b, and microcapsule in the field of view was opened, Fig. 10c. The

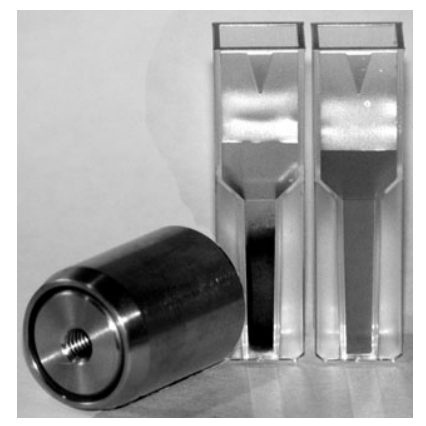

Fig. 9 Microcapsules suspension affected by magnetic field. Magnet was placed in front of the left cuvette. Change in suspension color is observed in the left cuvette. This fact can be explained by change of capsules distribution. Suspension color in the right cuvette remained unchanged. Any distribution of color was not observed which means that capsules distribution change in the left cuvette was caused by magnetic field. Magnet (GX06 NdFeBa constant magnet, IBS Magnet, Germany) was placed in front of the left cuvette.

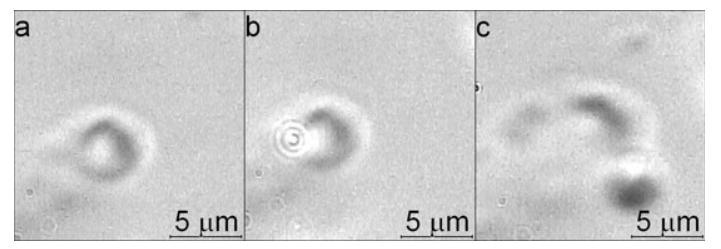

Fig. 10 Images of the capsule destruction process caused by the laser impulse: a - capsule before irradiation; b - capsule during irradiation; c - capsule after irradiation.

overall conclusion is that the concentration of the gold nanoparticles in the microcapsule shell was sufficient to make them sensitive to laser irradiation. We note that remote activation of microcapsules with controllable delivery described in this work is relevant for intracellular operations, ${ }^{22}$ and therefore potential applications of such a system are anticipated in bio-medicine.

\section{Conclusions}

We have fabricated multilayer capsules which are fully biocompatible and possess two functions: optically addressable and susceptible to magnetic field. Combination of two sorts of nanoparticles (magnetite and gold nanoparticles) in nanocomposite shells assembled by LbL resulted in tailoring different functions. Mutual interference of physical response while two sorts of nanoparticles were co-deposited was found to have negligible effect as compared with absolute amount of particles of each sort. A control over the motion of the microcapsules by using a constant magnet and also a sensitivity of the microcapsule shell to the laser irradiation for combined shells were shown to have the responsiveness properties fairly as whose one as for single component capsules. Nanoparticle arrangement in polymer based shells was different for magnetite and gold nanopartcles. The gold nanoparticles of $5 \mathrm{~nm}$ size were homogeneously distributed in the microcapsule shell, while heterogeneous distribution was observed for the iron oxide nanoparticles. The tendency of the iron oxide nanoparticles to agglomeration led to an enlargement of the insular structures in the microcapsule shell. Overall, the number of nanoparticles layers as deposited was found to be in incremental correlation with final amount of nanoparticles in final capsules as observed by electron microscopy. Gold nanoparticles of smaller size provide more dense and concentrated deposition across capsule surface when gold nanoparticles of bigger size.

In this research we demonstrate the biocompatibility of polyelectrolyte LbL capsules can be successfully combined with different physical functional properties brought by nanoparticles of different sorts assembled between the layers. Whilst tailored in one entity the long lasting demand of multifunctional delivery systems for biological application is to be fulfilled.

\section{Acknowledgements}

The work was supported by the BRIDGE Research UK-Russia cooperation project-RC10, the German-Russian cooperation project DFG436 RUS $113 / 844 / 0-1$ and RFBR 
(06-02-04009). The authors thank Matthieu Bedard for helpful discussions.

\section{References}

1 G. Decher, Science, 1997, 277, 1232-1237.

2 H. Möhwald, Colloids Surf., A, 2000, 171, 25-31.

3 G. B. Sukhorukov, E. Donath, S. Davis, H. Lichtenfeld, F. Caruso, V. I. Popov and H. Möhwald, Polym. Adv. Technol., 1998, 9, 759-767.

4 H. Ai, S. A. Jones, M. M. de Villiers and Y. M. Lvov, J. Controlled Release, 2003, 86, 59-68.

5 D. V. Andreeva, D. A. Gorin, H. Möhwald and G. B. Sukhorukov, Langmuir, 2007, 23, 9031-9036.

6 Q. Zhao, S. Zhang, W. Tong, C. Gao and J. Shen, Eur. Polym. J., 2006, 42, 3341-3351.

7 X. Teng, D. G. Shchukin and H. Möhwald, Adv. Funct. Mater., 2007, 17, 1273-1278.

8 E. Tjipto, K. D. Cadwell, J. F. Quinn, A. P. R. Johnston, N. L. Abbott and F. Caruso, Nano Lett., 2006, 6, 2243-2248.

9 G. A. Ozin and A. C. Arsenault, Nanochemistry (A Chemical Approach to Nanomaterials), Royal Society of Chemistry, 2005, Sun Fung Offset Binding Company Ltd, China, 2005, ch. 1, p. 628.

10 D. V. Andreeva, D. A. Gorin, D. G. Shchukin and G. B. Sukhorukov, Macromol. Rapid Commun., 2006, 27, 931-936.

11 W. F. Dong, G. B. Sukhorukov and H. Möhwald, Phys. Chem. Chem. Phys., 2003, 5, 3003-3012.

12 W. Song, M. Okamura, T. Kondoz and K. Uosaki, Phys. Chem. Chem. Phys., 2003, 5, 5279-5284.

13 D. Grigoriev, D. Gorin, G. B. Sukhorukov, A. Yashchenok, E. Maltseva and H. Möhwald, Langmuir, 2007, 23, 12388-12396.

14 A. Mamedov, J. Ostrander, F. Aliev and N. A. Kotov, Langmuir, 2000, 16, 3941-3949.

15 A. A. Mamedov and N. A. Kotov, Langmuir, 2000, 16, 5530-5533.

16 J. Cho, H. Jang, B. Yeom, H. Kim, R. Kim, S. Kim, K. Char and F. Caruso, Langmuir, 2006, 22, 1356-1364.

17 G. Sukhorukov, A. Fery, M. Brumen and H. Möhwald, Phys. Chem. Chem. Phys., 2004, 6, 4078-4089.

18 M. Bedard, A. G. Skirtach and G. B. Sukhorukov, Macromol. Rapid Commun., 2007, 28, 1517-1521.
19 A. G. Skirtach, A. A. Antipov, D. G. Shchukin and G. B. Sukhorukov, Langmuir, 2004, 20, 6988-6992.

20 B. Radt, N. F. Smith and F. Caruso, Adv. Mater., 2004, 16, 2184-2189.

21 A. G. Skirtach, C. Dejugnat, D. Braun, A. S. Susha, A. L. Rogach, W. J. Parak, H. Möhwald and G. B. Sukhorukov, Nano Lett., 2005, 5, 1371-1377.

22 A. G. Skirtach, A. M. Javier, O. Kreft, K. Köhler, A. P. Alberola, H. Möhwald, W. J. Parak and G. B. Sukhorukov, Angew. Chem., Int. Ed., 2006, 118, 4728-4733.

23 A. G. Skirtach, C. Dejugnat, D. Braun, A. S. Susha, A. L. Rogach and G. B. Sukhorukov, J. Phys. Chem. C, 2007, 111, 555-564.

24 Z. Lu, M. D. Prouty, Z. Guo, V. O. Golub, C. S. S. R. Kumar and Y. M. Lvov, Langmuir, 2005, 21, 2042-2050.

25 D. A. Gorin, D. G. Shchukin, A. I. Mikhailov, K. Köhler, S. A. Sergeev, S. A. Portnov, I. V. Taranov, V. V. Kislov and G. B. Sukhorukov, Tech. Phys. Lett., 2006, 32, 70-72.

26 D. G. Shchukin, D. A. Gorin and H. Möhwald, Langmuir, 2006, 22, 7400-7404.

27 A. G. Skirtach, B. G. De Geest, A. Mamedov, A. A. Antipov, N. A. Kotov and G. B. Sukhorukov, J. Mater. Chem., 2007, 17, 1050-1054.

28 B. G. De Geest, A. G. Skirtach, A. A. Mamedov, A. A. Antipov, N. A. Kotov, S. C. De Smedt and G. B. Sukhorukov, Small, 2007, 3, 804-808.

29 D. V. Volodkin, A. I. Petrov, M. Prevot and G. B. Sukhorukov, Langmuir, 2004, 20, 3398-3406.

30 S. A. Portnov, A. M. Yashchenok, A. S. Gubskii, D. A. Gorin, A. A. Neveshkin, B. N. Klimov, A. A. Nefedov and M. V. Lomova, Inst. Exp. Tech., 2006, 49, 849-854.

31 O. Kreft, A. G. Skirtach, G. B. Sukhorukov and H. Möhwald, Adv. Mater., 2007, 19, 3142-3145.

32 K. Sato, I. Suzuki and J. Anzai, Langmuir, 2003, 19, 7406-7412.

33 M. Fang, P. Grant, M. McShane, G. B. Sukhorukov, V. O. Golub and Y. M. Lvov, Langmuir, 2002, 18, 6338-6344.

34 M. Kolasinska and P. Warszynski, Bioelectrochemistry, 2005, 66, 65-70.

35 M. Kolasinska and P. Warszynski, Appl. Surf. Sci., 2005, 252, 759-765.

36 A. M. Javier, O. Kreft, A. P. Alberola, C. Kirchner, B. Zebli, A. S. Susha, E. Horn, S. Kempter, A. G. Skirtach, A. L. Rogach, J. Radler, G. B. Sukhorukov, M. Benoit and W. J. Parak, Small, 2006, 2, 394-400. 